PROCEEDINGS OF THE

AMERICAN MATHEMATICAL SOCIETY

Volume 124, Number 6, June 1996

\title{
UNIONS OF LOEB NULLSETS
}

\author{
DAVID A. ROSS \\ (Communicated by Andreas Blass)
}

\begin{abstract}
The union of every point-finite, completely measurable family of Loeb nullsets is itself a Loeb nullset, provided the nonstandard model satisfies a simple set-theoretic condition. One application of this result is that every Loeb measurable function into a metric space has a lifting.
\end{abstract}

\section{INTRODUCTION}

Recall Lusin's Theorem:

Let $f$ be a measurable function from a Radon probability space $(X, \mathcal{B}, P)$ to a second countable topological space. Then for every $\epsilon>0$ there is a compact $K \subseteq X$ such that the restriction of $f$ to $K$ is continuous and $P(K)>1-\epsilon$.

(By Radon we mean that $X$ is topological, $\mathcal{B}$ contains all Borel sets, and $P$ is compact-inner-regular, that is, $P(E)=\sup \{P(K) \mid K \subseteq E, K$ compact $\}$ for every $E \in \mathcal{B}$.)

More generally, a function $f$ from a topological probability space $(X, \mathcal{B}, P)$ to a topological space $Y$ is Lusin measurable provided for every $\epsilon>0$ there is a compact $K \subseteq X$ such that the restriction of $f$ to $K$ is continuous and $P(K)>1-\epsilon$. Fremlin $[\mathrm{F}]$ has proved that if $(X, \mathcal{B}, P)$ is Radon and $Y$ is metric, then $f$ is Lusin measurable.

A corresponding result to Lusin's Theorem in nonstandard measure theory is due originally to Robert Anderson ([SB], Theorem 2.1.4):

Every measurable function from a Loeb probability space to a second countable topological space has a lifting.

The correspondence is made precise in [R2], where it is proved that a function is Lusin measurable if and only if it admits a "two-legged" lifting. In addition, necessary and sufficient conditions for the existence of a lifting are given there; this yields a new sufficient condition for a function to be Lusin measurable.

Prikry and Kupka show in $[\mathrm{KuP}]$ that Fremlin's result follows easily once one knows that every point-finite, completely measurable family of Radon nullsets is a Radon nullset (see below). Prikry and Koumoullis [KoP] have extended this latter result to arbitrary compact probability spaces. (See $\S 2$ for definitions.) An analogous result about unions of Loeb nullsets, and a corresponding generalization of Anderson's theorem to general metric spaces, would be an immediate consequence of the standard theory, provided Loeb measures were compact.

Received by the editors July 6, 1993 and, in revised form, December 30, 1994.

1991 Mathematics Subject Classification. Primary 28E05; Secondary 03H05, 26E35.

Key words and phrases. Loeb measure, nonstandard analysis, compact measure, Loeb nullset. 
In [R1] it is shown that every compact probability space is the image of a Loeb space under a measurable map (Lemma 5.1, below); this suggests that Loeb spaces might indeed be compact. However, in a recent paper J. Aldaz [A] gives an example of a Loeb space which is not compact, rendering both the unions-of-nullsets result and the corresponding lifting result nontrivial for Loeb spaces.

This paper gives proofs for both these results. In fact, the unions-of-Loeb-nullsets result is strictly stronger than the corresponding standard result (see Corollary 5.2 , below). (Weaker results, requiring the much stronger hypothesis that the nonstandard model is "fully" saturated, appeared in [R3].)

\section{Preliminaries}

For $X$ a set, write $\mathcal{P}(X)$ for the power set of $X, \mathcal{P}_{n}(X)$ for $\{E \in \mathcal{P}(X) \mid \operatorname{card}(E)=$ $n\}$, and $\mathcal{P}_{\text {fin }}(X)$ for $\{E \in \mathcal{P}(X) \mid E$ finite $\}=\bigcup_{n=0}^{\infty} \mathcal{P}_{n}(X)$.

Suppose $\mathcal{F} \subseteq \mathcal{P}(X)$. $\mathcal{F}$ is point-finite if for every $x \in X,\{E \in \mathcal{F} \mid x \in E\}$ is finite. $\mathcal{F}$ has the finite intersection property if $E_{1} \cap \cdots \cap E_{n} \neq \emptyset$ whenever $\left\{E_{1}, \cdots, E_{n}\right\} \subseteq \mathcal{F}, n \in \mathbb{N}$. $\mathcal{F}$ is compact provided that for every subfamily $\mathcal{K} \subseteq \mathcal{F}$ with the finite intersection property, $\cap \mathcal{K} \neq \emptyset$.

Suppose now that $(X, \mathcal{B}, P)$ is a probability space. A family $\mathcal{F} \subseteq \mathcal{B}$ is completely measurable provided $\bigcup \mathcal{K} \in \mathcal{B}$ whenever $\mathcal{K} \subseteq \mathcal{F}$.

The probability space $(X, \mathcal{B}, P)$ is compact provided $P$ is inner-regular with respect to a compact family $\mathcal{K} \subseteq \mathcal{B}$.

The reader is assumed to be familiar with nonstandard analysis in general, and Loeb measures in particular, and is referred to $[\mathrm{C}]$ or $[\mathrm{SB}]$ for background. All nonstandard models will be assumed $\aleph_{1}$-saturated.

Suppose that $X$ is internal and $\mathcal{A} \subseteq{ }^{*} \mathcal{P}(X)$. Call $\mathcal{A} \kappa$-terraced provided (i) $\kappa=\operatorname{card}(\mathcal{A})$, and (ii) For some nondecreasing sequence $\left\{\mathcal{A}_{i}\right\}_{i<\kappa}$ with each $\mathcal{A}_{i} \subseteq \mathcal{A}$ compact, $\mathcal{A}=\bigcup_{i<\kappa} \mathcal{A}_{i}$. For $A \in \mathcal{A}$ let $\operatorname{Level}(A)=\min \left\{i<\kappa \mid A \in \mathcal{A}_{i}\right\}$. (Note this definition depends on choice of terracing; a particular one will always be assumed already chosen.)

Suppose $\alpha$ and $\kappa$ are cardinals; say that $\alpha$ is not measurably cofinal in $\kappa$, $\operatorname{NMC}(\alpha, \kappa)$, if either $\alpha$ is not a measurable cardinal (see below) or $\alpha$ is not cofinal in $\kappa$. Note that $\operatorname{NMC}(\alpha, \kappa)$ holds for every $\alpha$ precisely when the cofinality of $\kappa$ is not measurable.

Lemma 2.1. There exist nonstandard models such that for every internal $X$ and every internal $\mathcal{A} \subseteq{ }^{*} \mathcal{P}(X)$ with $\kappa=\operatorname{card}(\mathcal{A})$ infinite, $\mathcal{A}$ is $\kappa$-terraced and $\operatorname{NMC}(\alpha, \kappa)$ for all $\alpha$.

Proof. Suppose the nonstandard model satisfies the special model axiom $S M A_{\beta}$ (see $[\mathrm{R} 4]$ or $[\mathrm{J}]$ ). This means that for any first-order language $\mathcal{L}$ with fewer than $\beta$ relation, function, and constant symbols, and every $\mathcal{L}$-model $\mathfrak{A}=(A, \cdots)$ with both $A$ and every interpretation of every relation and function symbol internal, $\mathfrak{A}$ is a special model (see [CK] for definitions). It is shown in [R4] that all internal infinite sets have the same external cardinality $\kappa$ when $S M A_{\beta}$ holds, $\beta \geq \aleph_{0}$.

Suppose that $X$ and $\mathcal{A} \subseteq{ }^{*} \mathcal{P}(X)$ are internal, and that $\mathcal{A}$ is infinite. Let $\mathcal{L}$ be the first-order language with unary predicate symbol $Q(x)$ and binary predicate $E(x, y)$, and let $\mathfrak{A}=(A, \cdots)$ be the $\mathcal{L}$-structure with $A=X \cup \mathcal{A}, Q^{\mathfrak{A}}=\mathcal{A}$, and $E^{\mathfrak{A}}=\{(x, y) \in X \times \mathcal{A} \mid x \in y\}$. By $S M A_{\beta}$, there is an elementary chain $\mathfrak{A}_{0} \preceq$ 
$\mathfrak{A}_{1} \preceq \cdots$ with $\mathfrak{A}_{i} i^{+}$-saturated and $\mathfrak{A}=\bigcup_{i<\kappa} \mathfrak{A}_{i}$. Let $\left\{a_{i}\right\}_{i<\kappa}$ be any enumeration of $\mathcal{A}$, and put $\mathcal{A}_{i}=Q^{\mathfrak{A}} \cap\left\{a_{j}\right\}_{j \leq i}$. Evidently $\mathcal{A}=\bigcup_{i<\kappa} \mathcal{A}_{i}$. It remains to show that $\mathcal{A}_{i}$ is compact.

Suppose $\mathcal{K} \subseteq \mathcal{A}_{i}$ has the finite intersection property. Consider the set $\Gamma(x)$ of $\mathcal{L} \cup A_{i}$-formulas of form $E(x, K), K \in \mathcal{K}$. This set has cardinality $\leq i$, and since $\mathcal{K}$ has the finite intersection property, $\Gamma(x)$ is finitely consistent, so by $i^{+}$-saturation of $\mathfrak{A}_{i}$ there is an $a \in A_{i}$ with $\mathfrak{A}_{i} \models E(a, K)$ for all $K \in \mathcal{K}$, i.e., $a \in \cap \mathcal{K}$, proving compactness.

Thus any nonstandard model where (i) $S M A_{\beta}$ holds for some $\beta \geq \aleph_{0}$, and (ii) $\kappa$ is not cofinally measurable (for example, $\kappa$ regular and not measurable) will work; this proves the lemma.

Suppose that $(\Omega, \mathcal{A}, \mu)$ is an internal, ${ }^{*}$ finitely-additive probability space. Denote by $\left(\Omega, \mathcal{A}_{L}, \mu_{L}\right)$ the (standard) probability space generated from $(\Omega, \mathcal{A}, \mu)$ by the Loeb construction. Recall that a measurable function $f$ from $\Omega$ to a topological space $Y$ has a lifting $F$ provided $F: \Omega \longrightarrow^{*} Y$ is internal and $F(\omega) \approx f(\omega)$ $\mu_{L}$-almost everywhere.

The main results can now be stated; the proofs are deferred to $\S 4$.

Theorem 2.2. Let $(\Omega, \mathcal{A}, \mu)$ be an internal ${ }^{*}$ finitely-additive probability space, and let $\mathcal{E}=\left\{A_{i}\right\}_{i<\alpha}$ be a point-finite, completely measurable family of $\mu_{L}$-nullsets. Suppose that $\mathcal{A}$ is $\kappa$-terraced, and that $\operatorname{NMC}(\alpha, \kappa)$ holds. Then $\mu_{L}\left(\bigcup_{i<\alpha} A_{i}\right)=0$.

Corollary 2.3. Suppose $(\Omega, \mathcal{A}, \mu)$ is an internal ${ }^{*}$ finitely-additive probability space, where $\mathcal{A}$ is $\kappa$-terraced, and that $\operatorname{NMC}(\alpha, \kappa)$ holds for all $\alpha$. Let $f$ be a measurable function from $\Omega$ to a metric space $Y$. Then for some $\Omega^{\prime} \subseteq \Omega$ with $\mu_{L}\left(\Omega^{\prime}\right)=1$, the restriction of $f$ to $\Omega^{\prime}$ has second countable range (and so $f$ has a lifting, by Anderson's lifting theorem).

\section{Useful STANDARD LEMMAS}

The first lemma is due to Bernstein; see ([KuP], Proposition 3.5) for a proof.

Lemma 3.1. There is a subset $\mathbb{B}$ of $[0,1]$ with $\operatorname{card}(\mathbb{B})=2^{\aleph_{0}}$ such that every closed subset of $\mathbb{B}$ is at most countable.

Let $X$ be an arbitrary set, and $\nu: \mathcal{P}(X) \longrightarrow[0, \infty)$. A set $E \in \mathcal{P}(X)$ is an atom for $\nu$ if $\nu(E) \neq 0$ and $\{\nu(B), \nu(E-B)\}=\{0, \nu(E)\}$ for every $B \subseteq E$. If $\nu$ has no atoms it is atomless. If $\alpha$ is an infinite cardinal and $\nu$ is a finite measure on $(\alpha, \mathcal{P}(\alpha))$ for which (i) $\alpha$ is an atom, (ii) singletons are nullsets, and (iii) $\nu(\bigcup \mathcal{E})=0$ whenever $\mathcal{E}$ is a family of nullsets with $\operatorname{card}(\mathcal{E})<\alpha$, then $\alpha$ is a measurable cardinal.

The following is a form of Ramsey's Theorem due to Hajnal and Erdös; see [K] for a proof.

Lemma 3.2. If $\alpha$ is a measurable cardinal and $\varphi: \mathcal{P}_{\text {fin }}(\alpha) \longrightarrow\{0,1\}$, then there is a subset $\widehat{\alpha} \subseteq \alpha$ such that $\operatorname{card}(\widehat{\alpha})=\alpha$ and such that $\varphi$ is constant on $\mathcal{P}_{n}(\widehat{\alpha})$ for every $n$.

The final lemma in this section is ([KoP], Lemma 4). 
Lemma 3.3. If $\mathcal{F}$ is an uncountable family of nonnullsets in a finite measure space, then for some $x,\{A \in \mathcal{F} \mid x \in A\}$ is infinite. (In other words, there is no point-finite uncountable family of nonnullsets in a finite measure space.)

\section{Proof of main Results}

Proof of Theorem 2.2. Otherwise, let $\mathcal{E}=\left\{A_{i}\right\}_{i<\alpha}$ be a counterexample, that is, a point-finite completely measurable family of Loeb nullsets with $\mu_{L}\left(\bigcup_{i<\alpha} A_{i}\right)>0$.

Without loss of generality, $\alpha$ has the least cardinality of any counterexample, and $\mu_{L}\left(\bigcup_{i<\alpha} A_{i}\right)=1$. Put $A=\bigcup_{i<\alpha} A_{i}$.

For $E \subseteq \alpha$ put $\nu(E)=\mu_{L}\left(\bigcup_{i \in E} A_{i}\right)$. Consider two cases:

Case 1. $\nu$ is atomless on $(\alpha, \mathcal{P}(\alpha))$. Inductively define partitions $P_{\lambda}, \lambda<\omega_{1}$, of $\alpha$ as follows. Put $P_{0}=\{\alpha\}$. If $\beta<\omega_{1}$ is a limit ordinal, let $P_{\beta}$ consist of all nonempty intersections of the form $\bigcap_{\lambda<\beta} E_{\lambda}$, where $E_{\lambda} \in P_{\lambda}$ for all $\lambda<\beta$. Given $P_{\lambda}$, construct $P_{\lambda+1}$ by replacing each $E$ in $P_{\lambda}$ by itself if $\nu(E)=0$, otherwise by disjoint sets $E_{1}, E_{2}$ where $E_{1} \cup E_{2}=E$ and $0<\nu\left(E_{1}\right) \leq \nu\left(E_{2}\right) \leq \nu(E)$. (We may take $\nu\left(E_{1}\right) \neq 0$ since from the definition of $\nu, \nu\left(E_{1} \cup E_{2}\right) \leq \nu\left(E_{1}\right)+\nu\left(E_{2}\right)$.)

Let $\alpha=E_{0} \supseteq E_{1} \supseteq E_{2} \supseteq \cdots$, with $E_{\lambda} \in P_{\lambda}$ for all $\lambda<\omega_{1}$. (Call such a sequence adapted.) Suppose (for a contradiction) that $\nu\left(E_{\lambda}\right) \neq 0$ for every $\lambda$. Put $B_{\lambda}=E_{\lambda} \backslash E_{\lambda+1}$. By the construction, $\nu\left(B_{\lambda}\right)>0$ for every $\lambda$; but then $\left\{\bigcup_{i \in B_{\lambda}} A_{i}\right\}_{\lambda<\omega_{1}}$ is a point-finite uncountable collection of nonnull sets, contradicting Lemma 3.3.

It follows that every adapted sequence is eventually constant, so there are at most $\aleph_{0}$ of them. Let $\left\{E_{\tau}\right\}_{\tau \in T}$ be the set of intersections of adapted sequences; we may take $T \subseteq \mathbb{B}$. For $\tau \in T$ put $A_{\tau}=\bigcup_{i \in E_{\tau}} A_{i}$; note that $\mu_{L}\left(A_{\tau}\right)=\nu\left(E_{\tau}\right)=0$, and that $\left\{E_{\tau}\right\}_{\tau \in T}$ forms a partition of $\alpha$.

For $x \in A$ let $f(x)$ be the smallest (in the sense of the ordering on $\mathbb{B}$ inherited from $\mathbb{R})$ of the finitely many $\tau$ with $x \in A_{\tau}$. Note that for $0 \leq r \leq 1, f^{-1}([0, r))=$ $\bigcup_{\tau<r} A_{\tau}$, so $f: A \longrightarrow[0,1]$ is Loeb measurable.

It follows that $f$ has a lifting, and for some internal $K \subseteq A$ with $\mu_{L}(K)>0$ and some internal $F: K \longrightarrow^{*}[0,1], F(x) \approx f(x)$ for all $x \in K$. Since $f(K)=\operatorname{st}(F(K))$ is closed ([SB], Proposition 2.1.1) and $f(K) \subseteq \mathbb{B}, f(K)$ is at most countable, and

$$
\begin{aligned}
0 & <\mu_{L}(K) \\
& \leq \mu_{L}\left(f^{-1}(f(K))\right) \\
& =\mu_{L}\left(\bigcup_{\tau \in f(K)} f^{-1}(\tau)\right) \\
& \leq \mu_{L}\left(\bigcup_{\tau \in f(K)} A_{\tau}\right) \quad\left(\text { since } f(x)=\tau \text { only if } x \in A_{\tau}\right) \\
& =0 \quad\left(\text { since } f(K) \text { countable and } \mu_{L}\left(A_{\tau}\right)=0\right) .
\end{aligned}
$$

This is a contradiction, and proves the theorem in this case. 
Case 2. $\nu$ has an atom in $(\alpha, \mathcal{P}(\alpha))$. Without loss of generality, $\alpha$ is the atom. By minimality of $\alpha$, if $E \subseteq \alpha$ and $\operatorname{card}(E)<\alpha$, then $\nu(E)=0$. It is easy to verify in this case that $\nu$ is actually a measure, so $\alpha$ is a measurable cardinal.

For each $\lambda<\alpha$, put $B_{\lambda}=\bigcup_{i>\lambda} A_{i}$. Note $\mu_{L}\left(B_{\lambda}\right)>0$, so there is an internal $K_{\lambda} \subseteq B_{\lambda}$ with $\mu_{L}\left(K_{\lambda}\right)>0$. Choose this $K_{\lambda}$ so that $\operatorname{Level}\left(K_{\lambda}\right)$ is minimal.

Define $\varphi: \mathcal{P}_{\text {fin }}(\alpha) \longrightarrow\{0,1\}$ by

$$
\varphi(E)= \begin{cases}1 & \text { if } E=\emptyset \text { or } \\ 0 & \text { otherwise. }\end{cases}
$$

By Theorem 3.2 there is a subset $\widehat{\alpha} \subseteq \alpha, \operatorname{card}(\widehat{\alpha})=\alpha$, such that $\varphi$ is constant on each $\mathcal{P}_{n}(\widehat{\alpha})$. By Lemma $3.3, \varphi \equiv 1$ on $\mathcal{P}_{n}(\widehat{\alpha})$ whenever $n>1$, so $\left\{K_{\lambda}\right\}_{\lambda \in \widehat{\alpha}}$ has the finite intersection property.

Let $\mathcal{O}: \widehat{\alpha} \longrightarrow \kappa$ be the map $\mathcal{O}(\lambda)=\operatorname{Level}\left(K_{\lambda}\right)$. Since $B_{\lambda}$ decreases in $\lambda$ and $K_{\lambda}$ is chosen to minimize $\operatorname{Level}\left(K_{\lambda}\right)$ for each $\lambda, \mathcal{O}$ is nondecreasing on $\widehat{\alpha}$.

Define a function $\varphi_{1}: \mathcal{P}_{\text {fin }}(\widehat{\alpha}) \longrightarrow\{0,1\}$ by

$$
\varphi_{1}(E)= \begin{cases}1 & \text { if } \mathcal{O}\left(\lambda_{1}\right)=\mathcal{O}\left(\lambda_{2}\right) \forall \lambda_{1}, \lambda_{2} \in E, \\ 0 & \text { otherwise. }\end{cases}
$$

Applying Theorem 3.2 again, there is $\hat{\hat{\alpha}} \subseteq \widehat{\alpha}$ with $\operatorname{card}(\hat{\hat{\alpha}})=\alpha$ and $\varphi_{1}$ constant on $\mathcal{P}_{n}(\hat{\hat{\alpha}})$ for every $n$.

Suppose (for a contradiction) that $\varphi_{1} \equiv 1$ on $\mathcal{P}_{2}(\hat{\hat{\alpha}})$; then for some $\gamma<\kappa$, $\gamma=\mathcal{O}(\lambda)=\operatorname{Level}\left(K_{\lambda}\right)$ for all $\lambda \in \hat{\hat{\alpha}}$. Since $\left\{K_{\lambda}\right\}_{\lambda \in \hat{\alpha}}$ has the finite intersection property and $\left\{K \in{ }^{*} \mathcal{P}(\Omega) \mid \operatorname{Level}(K)=\gamma\right\}$ is a compact family, there is an $x \in \bigcap_{\lambda \in \hat{\hat{\alpha}}} K_{\lambda}$. By point-finiteness of $\left\{A_{i}\right\}_{i<\alpha}$, there is a largest $\beta<\alpha$ with $x \in A_{\beta}$. Let $\lambda \in \hat{\hat{\alpha}}, \lambda>\beta$; then $x \notin \bigcup_{i \geq \lambda} A_{i}=B_{\lambda}$, but $x \in K_{\lambda} \subseteq B_{\lambda}$, a contradiction.

Therefore, $\varphi_{1} \equiv 0$ on $\mathcal{P}_{2}(\hat{\hat{\alpha}})$, so $\mathcal{O}$ is strictly increasing on $\hat{\hat{\alpha}}$. The argument used in the last paragraph shows that $\{\mathcal{O}(\lambda)\}_{\lambda \in \hat{\hat{\alpha}}}$ is unbounded in $\kappa$, so $\hat{\hat{\alpha}}$, hence $\alpha$, is cofinal in $\kappa$, contradicting the hypothesis on $\kappa$. The theorem is proved.

Proof of Corollary 2.3. By paracompactness of $Y$, for every $n \in \mathbb{Z}^{+}$there is a pointfinite open cover $\mathcal{U}_{n}$ of $Y$ by $\frac{1}{n}$-balls. By Lemma 3.3, we can write $\mathcal{U}_{n}=\mathcal{U}_{n}^{1} \cup \mathcal{U}_{n}^{2}$, where (i) $\mathcal{U}_{n}^{1}$ is countable, (ii) $\mathcal{U}_{n}^{1} \cap \mathcal{U}_{n}^{2}=\emptyset$, and (iii) for every $u \in \mathcal{U}_{n}^{2}, f^{-1}(u)$ is a $\mu_{L}$-nullset. Put $\Omega_{n}=f^{-1}\left(\bigcup \mathcal{U}_{n}^{1}\right)$; by Theorem $2.2, f^{-1}\left(\Omega \backslash \Omega_{n}\right) \subseteq \bigcup_{\mu \in \mathcal{U}_{n}^{2}} f^{-1}(\mu)$ is a $\mu_{L}-$ nullset. Put $\Omega^{\prime}=\bigcap_{n} \Omega_{n}$; it is easy to confirm that $\Omega^{\prime}$ satisfies the conditions of the lemma.

\section{A STANDARD COROLLARY}

Lemma 5.1. Let $(X, \mathcal{B}, P)$ be a (standard) compact probability space, where the nonstandard model is $\operatorname{card}(\mathcal{B})^{+}$-saturated. Then there is a Loeb space $\left(\Omega, \mathcal{A}_{L}, \mu_{L}\right)$ and a measurable function $\varphi: \Omega \longrightarrow X$, so that $P=\mu_{L} \circ \varphi^{-1}$.

Proof. See $[\mathrm{R} 1]$. 
Corollary 5.2. Let $(X, \mathcal{B}, P)$ be a compact probability space, and $\left\{A_{i}\right\}_{i<\alpha}$ a pointfinite completely measurable family of $P$-nullsets. Then $P\left(\bigcup_{i<\alpha} A_{i}\right)=0$.

Proof. There exist nonstandard models satisfying $\mathrm{SMA}_{\beta}$ for $\beta$ arbitrarily large; it follows from Lemma 2.1 that there is a nonstandard model which is $\operatorname{card}(\mathcal{B})^{+}$ saturated, and for some $\kappa$ every internal family of internal sets is $\kappa$-terraced and satisfies $\operatorname{NMC}(\alpha, \kappa)$. Let $\left(\Omega, \mathcal{A}_{L}, \mu_{L}\right)$ and $\varphi: \Omega \longrightarrow X$ be as given in Lemma 5.1. Then $\left\{\varphi^{-1}\left(A_{i}\right)\right\}_{i<\alpha}$ is a point-finite completely measurable family of $\mu_{L}$-nullsets, so $P\left(\bigcup_{i<\alpha} A_{i}\right)=\mu_{L}\left(\varphi^{-1}\left(\bigcup_{i<\alpha} A_{i}\right)\right)=\mu_{L}\left(\bigcup_{i<\alpha} \varphi^{-1}\left(A_{i}\right)\right)=0$ by Theorem 2.2 .

\section{ACKNOWLEDGEMENTS}

The author is grateful to Dale Myers, Jerry Keisler, and the referee for pointing out errors in earlier drafts.

\section{REFERENCES}

[A] J. Aldaz, Compactness and Loeb measures (to appear).

[C] N. Cutland (ed.), Nonstandard Analysis and its Applications, Cambridge University Press, Cambridge, England, 1988. MR 89m:03060

[CK] C.C. Chang and H.J. Keisler, Model Theory, North Holland, Amsterdam, The Netherlands, 1973. MR 53:12927

[F] D. H. Fremlin, Measurable functions and almost continuous functions, Manuscripta Math. 33 (1981), 387-405. MR 82e:28006

$[\mathrm{J}] \quad$ R. Jin, The isomorphism property versus the special model axiom, J. Symbolic Logic $\mathbf{5 7}$ (1992), 975-987. MR 94c:03079

$[\mathrm{K}] \quad$ E. M. Kleinberg, Infinitary combinatorics, Cambridge Summer School in Mathematical Logic, Lecture Notes in Math., Vol. 337, Springer, Berlin, 1973, pp. 361-418. MR 49:2400

[KoP] G. Koumoullis and K. Prikry, The Ramsey property and measurable selections, J. Lond. Math. Soc. 28 (1983), 203-210. MR 85g:54010

[KuP] J. Kupka and K. Prikry, The measurability of uncountable unions, Amer. Math. Monthly 91 (1984), 85-97. MR 85g:28015

[R1] D. A. Ross, Compact measures have Loeb preimages, Proc. Amer. Math. Soc. 115 (1992), 365-370. MR 92i:28023

[R2] L Lifting theorems in nonstandard measure theory, Proc. Amer. Math. Soc. 109 (1990), 809-822. MR 91b:03110

[R3] , Measurable Transformations in Saturated Models of Analysis, Ph. D. Thesis, Univ. of Wisconsin-Madison, 1983.

[R4] The special model axiom in nonstandard analysis, J. Symbolic Logic $\mathbf{5 5}$ (1990), 1233-1242. MR 91h:03091

[SB] K. D. Stroyan and J. M. Bayod, Foundations of Infinitesimal Stochastic Analysis, North Holland / Elsevier Science Publishers, Amsterdam, The Netherlands, 1986. MR 87m:60001

Department of Mathematics, University of Hawail at Manoa, Honolulu, Hawail 96822

E-mail address: ross@math.hawaii.edu 